Journal for ImmunoTherapy of Cancer

\section{Incomplete Freund's adjuvant reduces arginase and enhances Th1 dominance, TLR signaling and CD40 ligand expression in the vaccine site microenvironment}

To cite: Pollack KE, Meneveau M0, Melssen MM, et al. Incomplete Freund's adjuvant reduces arginase and enhances Th1 dominance, TLR signaling and CD40 ligand expression in the vaccine site microenvironment. Journal for ImmunoTherapy of Cancer 2020;8:e000544. doi:10.1136/ jitc-2020-000544

- Additional material is published online only. To view, please visit the journal online (http://dx.doi.org/10.1136/jitc2020-000544).

Society of Immunotherapy of Cancer Annual Meeting, Nov. 2019, National Harbor, MD

KEP and MOM are joint first authors.

Accepted 30 March 2020

Check for updates

(C) Author(s) (or their employer(s)) 2020. Re-use permitted under CC BY. Published by BMJ.

For numbered affiliations see end of article.

Correspondence to Dr Craig L Slingluff Jr; CLS8H@virginia.edu

\section{ABSTRACT}

Background Immunogenicity of cancer vaccines is impacted by adjuvants and schedule, but systematic assessments of their effects have not been performed. Montanide ISA-51, an incomplete Freund's adjuvant (IFA), is used in many vaccine trials, but concerns have been raised about negative effects in murine studies. We found in humans that IFA enhances systemic immune responses and that repeat vaccination at one site (same site vaccination (SSV)) creates tertiary lymphoid structures (TLS) in the vaccine site microenvironment (VSME). We hypothesized that vaccination with peptides+IFA+pICLC or $\mathrm{SSV} \times 3$ with peptides in IFA would create an immunogenic milieu locally at the VSME, with activated dendritic cells (DC), TLS-associated chemokines and a Th1-dominant VSME.

Methods Biopsies of the VSME were obtained from participants on two clinical trials who were immunized with multiple melanoma peptides (MELITAC 12.1) in adjuvants comprising IFA and/or the TLR3-agonist pICLC. Biopsies were obtained either a week after one vaccine or a week after $\mathrm{SSV} \times 3$. Controls included normal skin and skin injected with IFA without peptides. Gene expression analysis was performed by RNAseq.

Results VSME samples were evaluated from 27 patients. One vaccine with peptides in pICLC+IFA enhanced expression of CD80, CD83, CD86 ( $p<0.01), C D 40$ and CD40L $(p<0.0001)$ over normal skin; these effects were significantly enhanced for SSV with peptides+IFA. Vaccines containing pICLC increased expression of TBX21 (T-bet) but did not decrease GATA3 over normal skin, whereas SSV with peptides in IFA dramatically enhanced TBX21 and decreased GATA3, with high expression of IFN $\gamma$ and STAT1. SSV with peptides in IFA also reduced arginase-1 (ARG1) expression and enhanced expression of TLR adapter molecules TICAM-1 (TRIF) and MYD88. Furthermore, SSV with IFA and peptides also enhanced expression of chemokines associated with TLS formation.

Conclusions These findings suggest that SSV with peptides in IFA enhances CD40L expression by CD4 T cells, supports a Th1 microenvironment, with accumulation of activated and mature DC. Increased expression of TLR adaptor proteins after SSV with peptides in IFA might implicate effects of the skin microbiome. Reduced ARG1 may reflect diminished suppressive myeloid activity in the VSME.

Trial registration number (NCT00705640, NCT01585350).

\section{BACKGROUND}

Cancer vaccines can expand immune responses to tumor antigens; however, their activity depends on an effective immunological adjuvant. Newer adjuvants include toll-like receptor (TLR) agonists, saponins, RNA and nanoparticles. ${ }^{1}$ More traditional adjuvants include incomplete Freund's adjuvant (IFA), administered as a water-in-oil emulsion. A more contemporary formulation of IFA, Montanide ISA-51 (Seppic, Paris, France), has better-characterized components than earlier formulations, with toxicity profiles similar to those of other promising adjuvants. ${ }^{2}$ IFA continues to be used in veterinary vaccines. ${ }^{3}$ Montanide ISA-51 emulsions have been used in humans in clinical trials of new influenza vaccines, ${ }^{4-8}$ and in a lung cancer vaccine licensed in Cuba. ${ }^{9}$ In prior studies, others and we have reported high rates of $\mathrm{CD}^{+}$and $\mathrm{CD} 4^{+}$T-cell responses to peptide vaccines for cancer administered in IFA emulsions. ${ }^{10-12}$ High rates of tumor regression have also been observed after vaccination with an HPV peptide vaccine using IFA as an immunological adjuvant. ${ }^{13}$ Thus, human experience supports the use of IFA as a vaccine adjuvant.

On the other hand, murine studies have raised concerns about IFA, showing better T-cell responses with a TLR agonist plus an agonistic CD40 antibody (Ab).${ }^{14}$ Considering 
this finding, we performed a clinical trial in humans, testing each of two TLR agonists, with or without IFA. An agonistic CD40 Ab was not available for use; so, as an alternative, the vaccine included an antigen that activates $\mathrm{CD} 4^{+}$ $\mathrm{T}$ cells ${ }^{1011}$ to induce $\mathrm{CD} 40 \mathrm{~L}$ and thus to activate CD40 on DC. In contrast to what might have been expected with the murine data, we found that adding IFA significantly enhanced both the magnitude and persistence of antigenspecific CD8 T-cell responses. ${ }^{15}$ Differences in the impact of IFA in the murine model versus in humans may be explained by differences in IFA dose, differences in T-cell reactivity or to species differences. IFA does support T-cell and antibody responses to peptide antigens in humans and remains a viable adjuvant for cancer vaccines.

However, remarkably little is known about the effects of IFA locally in the vaccine site microenvironment (VSME). In prior studies, we evaluated the VSME for immune cell infiltrates by immunohistochemistry and found dense aggregates of $\mathrm{T}$ cells many of which are retained $>6$ weeks after the last vaccine. ${ }^{16}{ }^{17}$ We also found by flow cytometry that $\mathrm{T}$ cells infiltrating the VSME induced with IFA and peptide represent higher proportions of total $\mathrm{T}$ cells than in the blood. On the other hand, we found that these cells were less functional in an ELIspot assay than circulating vaccine-induced $\mathrm{T}$ cells and that they overexpressed integrins that may mediate their retention in peripheral tissues. ${ }^{18}$ These findings, and others, raised concern that the VSME induced with IFA may not support a favorable T-cell response. However, the outcome of our Mel58 clinical trial showed that adding IFA to TLR agonists enhanced the frequency, magnitude, and persistence of T-cell responses. Thus, a goal of the present study was to assess whether expression of immune-related genes in the VSME of patients on that trial (Mel58) and one prior trial (Mel48) may explain the favorable immunogenicity associated with IFA in humans.

In addition to the selection of adjuvant, the timing and route of vaccination may impact immunogenicity. Prior work has shown that immunogenicity of peptide vaccines can be enhanced by increased frequency of vaccine administration. ${ }^{12}$ We have found that vaccines administered in the skin (half-intradermal, half-subcutaneous) induce T-cell responses in $80 \%-100 \%$ of patients, and that particularly strong T-cell responses were observed when vaccines were administered at the same site each week (same site vaccination (SSV)). ${ }^{1011} 19$ Interestingly, SSV with peptides and IFA can induce development of tertiary lymphoid structures (TLS) in the VSME. ${ }^{16}$ TLS in other tissue sites support ongoing immune responses to local antigens, and they contain mature dendritic cells (DC), aggregates of T and B cells and specialized vasculature resembling high endothelial venules, all of which have been induced in the VSME with SSV $\times 3$. DC activation and maturation are crucial for optimal antigen presentation, yet vaccine adjuvants have not been compared systematically for their ability to enhance DC activation in the VSME. Classically, these processes are supported by $\mathrm{CD} 40$ ligation, which is provided naturally by $\mathrm{CD} 40 \mathrm{~L}$ on activated CD4 T cells. ${ }^{20}{ }^{21}$ However, it is not known whether vaccines induce accumulation of $\mathrm{CD}^{+} \mathrm{CD}_{40 \mathrm{~L}^{+} \mathrm{T}}$ cells in the VSME where they can interact directly with DC to support their maturation. We hypothesized that CD40L expression and DC activation would be enhanced in the VSME after repeated immunization with peptides in IFA, as well as by addition of TLR agonists. Another goal of the present application was to assess whether the same conditions that induce TLS also would induce expression of chemokines implicated in TLS formation. ${ }^{22} 23$

In prior immunohistochemistry (IHC) studies of the VSME, we found early dominance of $\mathrm{GATA}^{+}$cells over Tbet $^{+}$cells after one vaccine with IFA, suggesting a Th2-dominant VSME, ${ }^{18}$ which arguably is not ideal for supporting T-cell responses. However, SSV increased Tbet ${ }^{+}$ cells (Th1). ${ }^{18}$ Those studies used only single-color IHC and, thus, were limited in scope. In the present study, we hypothesized that SSV $\times 3$ with IFA would enhance expression of genes associated with a Th1-dominant microenvironment. We also performed exploratory analyses of other changes in the VSME, and report evidence for TLR signaling and downregulation of arginase I expression with IFA SSV $\times 3$. This work is enabled by prior collection of VSME biopsies from patients on the Mel48 and Mel58 clinical trials, which collected tissue from the VSME at multiple time points. These findings should contribute to a better understanding of the effects of IFA and TLR agonists in the VSME.

\section{METHODS \\ Patients and trials}

Samples for this study were obtained from patients enrolled in the Mel48 and Mel58 clinical trials at the University of Virginia, for which primary immunological findings have been reported. ${ }^{15} 1718$ For both trials, each peptide vaccine MELITAC 12.1 consisted of $100 \mu \mathrm{g}$ of each of 12 melanoma peptides recognized by CD8 T cells $(12 \mathrm{MP})^{19}$ plus $200 \mu \mathrm{g}$ of a tetanus toxoid helper peptide (Tet Tet) ${ }^{24}$ Briefly, for Mel48, patients with resected stage IIB-IV melanoma were randomly assigned to two study groups, each with five subgroups. All participants were vaccinated with MELITAC 12.1 emulsified in IFA (Montanide ISA-51, Seppic) in one extremity, half-subcutaneously and half-intradermally, on days 1, 8, 15, 29, 36 and 43, along with a second immunization at a different skin site, where that immunization was either adjuvant only (group 1) or MELITAC 12.1+adjuvant (group 2). For the present study, we have vaccine site biopsies taken at week 0 (prevaccine: groups 1A, 2A), week 1 (groups 1B, 2B) or week 3 (groups 1C, 2C). In the Mel58 trial, eligible patients were vaccinated with MELITAC 12.1, mixed with either of two TLR agonists (polyICLC (Hiltonol, Oncovir, Washington, District of Columbia, USA_, or endotoxin (lipopolysaccharide (SAIC-Frederick, Frederick, Maryland, USA)) ), with or without IFA. ${ }^{15}$ Each immunization was administered on one skin location, and subsequent immunizations were given in different skin sites (rotating 
vaccine sites) on days $1,8,15,36,57$ and 78 . One week after the first vaccine, patients underwent biopsy of the vaccine site. For the present report, vaccine-site biopsies from patients immunized with the combination of MELITAC 12.1 and polyICLC $(1 \mathrm{mg})$, with and without IFA, were analyzed. All patients were studied following informed consent, and with Institutional Board Review (IRB) (HSR-IRB 13498 and 15781, respectively) and Food and Drug Administration approval.

\section{RNA extraction and library preparation}

Total RNA was isolated from cells collected at the vaccination sites detailed above. RNA extraction was performed using the RNeasy Lipid Tissue MiniKit (Qiagen), according to manufacturer's recommendations. RNA samples were processed for library preparation using the NEBNext Ultra II Directional RNA Library Prep Kit (Illumina, San Diego, California, USA), according to validated standard operating procedures established by the UVA School of Medicine's Genome Analysis and Technology Core. Briefly, total RNA was used to isolate mRNA, using NEBNext Poly(A) mRNA Magnetic Isolation Module (New England Biolabs, Ipswich, Massachusetts, USA), followed by fragmentation and first-strand and secondstrand cDNA synthesis and fragmentation, as recommended by the manufacturer. The resulting cDNA was end-repaired, adenylated and then subjected to sequence adapter ligation. The final purified libraries were quantified and sized using the Invitrogen Qubit 3 Fluorometer (ThermoFisher Scientific, Waltham, Massachusetts, USA) and Agilent Technologies 4200 TapeStation (Agilent Technologies, Santa Clara, California, USA).

\section{Next-generation sequencing run and QC}

RNA sequencing was performed by the UVA genomics core using the Illumina NextSeq $75 \mathrm{bp}$ High Output sequencing kit reagent cartridge in conjunction with the Illumina NextSeq 500 (Illumina; 75 cycle, single read sequencing), according to the standard manufacturerrecommended procedure. Samples were randomized into four groups and run sequentially on the Illumina NextSeq 500 for single-end sequencing. After transfer to the Illumina Base Space interface, the quality of the runs was assessed by the number of reads in millions passing filter and the per cent of indexed reads. All runs passed the rigorous Illumina run quality control with an average of $94 \%$ passing filter, $94 \%$ Q30, $38 \mathrm{~Gb}$ per run, 439 million reads per run and $96 \%$ index recovery.

\section{Multiparameter immunofluorescence histology}

To test whether CD40L protein was present on $\mathrm{CD} 4 \mathrm{~T}$ cells, multiparameter immunofluorescence histology analysis was performed; $4 \mu \mathrm{m}$ thick sections were cut from formalin-fixed paraffin-embedded vaccine site tissue specimens, and human lymph node was used as a positive control. Multispectral staining was performed according to the manufacturer's protocol using the OPAL Multiplex Manual IHC kit, and antigen retrieval buffers (AR)
6 and 9 (Akoya Biosciences, Marlborough, Massachusetts, USA). Staining sequence, antibodies and antigen retrieval buffers were as follows:

AR9, CD40LG (1:50, Sigma Aldrich, St. Louis, Missouri, USA cat\#HPA045827) (Sigma Aldrich) Opal620; AR9, CD4 (1:100, clone SP35) (Cell Marque, Rocklin, California, USA) Opal520; AR9, CD8 (1:500, clone C8/144B) (Agilent Technologies) Opal540; AR6, ICOS1 (1:2k, clone D1K2T) (Cell Signaling) Opal650; AR6, CD20 (1:4k, clone L26) (Agilent Technologies) Opal570; AR9, CD3 (1:100, clone MRQ-39) (Cell Marque) Opal690 and spectral DAPI (Akoya Biosciences).

Stained slides were mounted using prolong diamond antifade (Life Technologies, Carlsbad, California, USA) and scanned at $10 \times$ magnification using the PerkinElmer Vectra 3.0 system and Vectra software (Akoya Biosciences). Regions of interest were selected in Phenochart software, and 20× magnification images were acquired with the Vectra 3.0 system. These images were spectrally unmixed using single stain positive control images in the InForm software (Akoya Biosciences). Images were analyzed using HALO software (Indica Labs, Albuquerque, New Mexico, USA).

\section{RNA sequencing and statistical analysis}

RNAseq reads were assessed for quality using FastQC. Transcript abundances were then quantified against the human reference genome (Gencode v28 Transcripts, Ensembl GRCh38) using Salmon ${ }^{25}$ and read into the R statistical computing environment as gene-level counts using the tximport package. ${ }^{26}$ The DESeq2 Bioconductor package $^{27} 28$ was used to normalize for differences in sequencing depth between samples (using the default median-of-ratios method), estimate dispersion and fit a negative binomial model for each gene. The BenjaminiHochberg False Discovery Rate procedure was then used to re-estimate the adjusted $p$ values. As an indirect validation of the RNAseq findings, linear regression was evaluated between previously published available IHC $\mathrm{data}^{17}$ from the vaccine sites and RNAseq data (online supplementary figure 1). All statistical analyses and data visualization, including GAGE, ${ }^{29} \mathrm{KEGG}^{30}$ and gene set enrichment analysis (GSEA) ${ }^{31}$ were done using the $\mathrm{R}$ statistical computing environment and GraphPad Prism 8 (GraphPad Software, San Diego, California, USA).

\section{RESULTS}

To assess effects of IFA and pICLC with and without peptides, we performed gene expression profiling on biopsies of vaccine sites obtained from 27 patients, including those enrolled in the Mel48 and Mel58 clinical trials (table 1).

\section{Dendritic cell activation and maturation}

Having noted formation of TLS that include mature DC after SSV vaccination with peptides in IFA, we hypothesized that this regimen would enhance expression of 
Table 1 Table 1Vaccine site treatment conditions by clinical trial

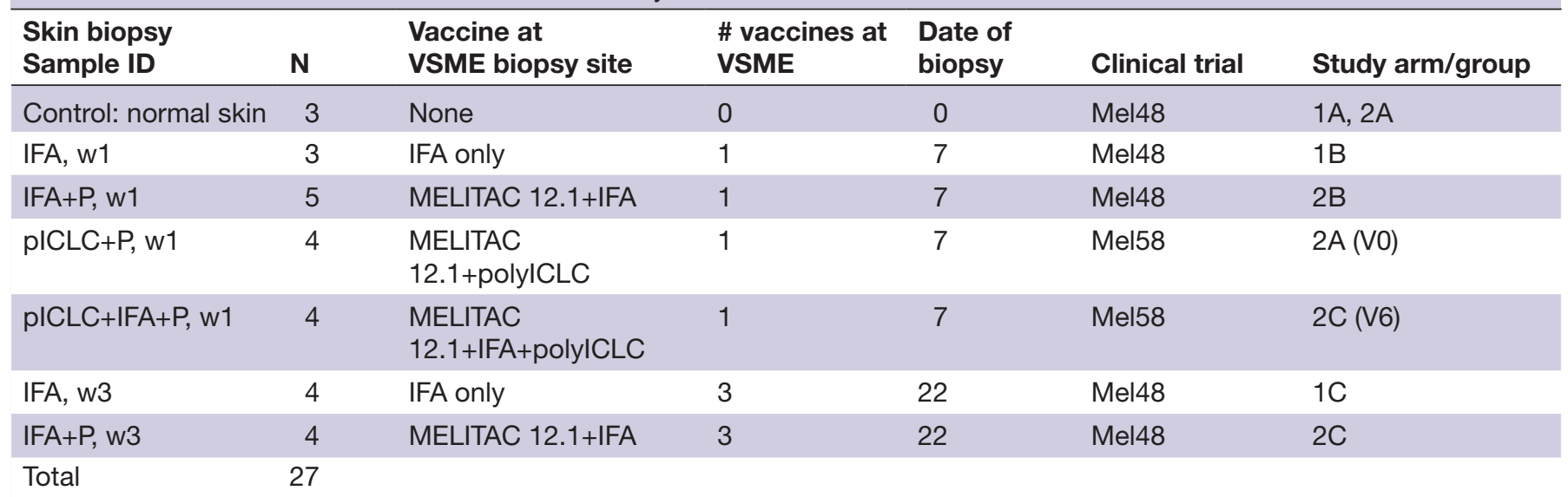

Number of evaluated samples with each vaccine site treatment condition.

IFA, incomplete Freund's adjuvant; $p$, peptide; pICLC, poly ICLC; w1, week 1; w3, week 3.

markers of DC activation (CD80, CD86) and maturation (CD83) in the VSME. One injection with IFA alone did not produce any significant changes in gene expression compared with normal skin (figure 1A-C). Adding peptides to IFA alone induced expression of CD80, CD83 and CD86, which trended non-significantly higher (figure 1A-C). After one vaccine with peptide+pICLC, expression of CD80 and CD83 were not significantly increased over normal skin, but CD86 expression was higher $(\mathrm{p}<0.01$, figure $1 \mathrm{C})$. Vaccines containing polyICLC+IFA+peptides generated a more robust increase in expression than either agent acting alone $(p<0.01 \mathrm{vs}$ control for CD80, CD83, CD86), suggesting that the

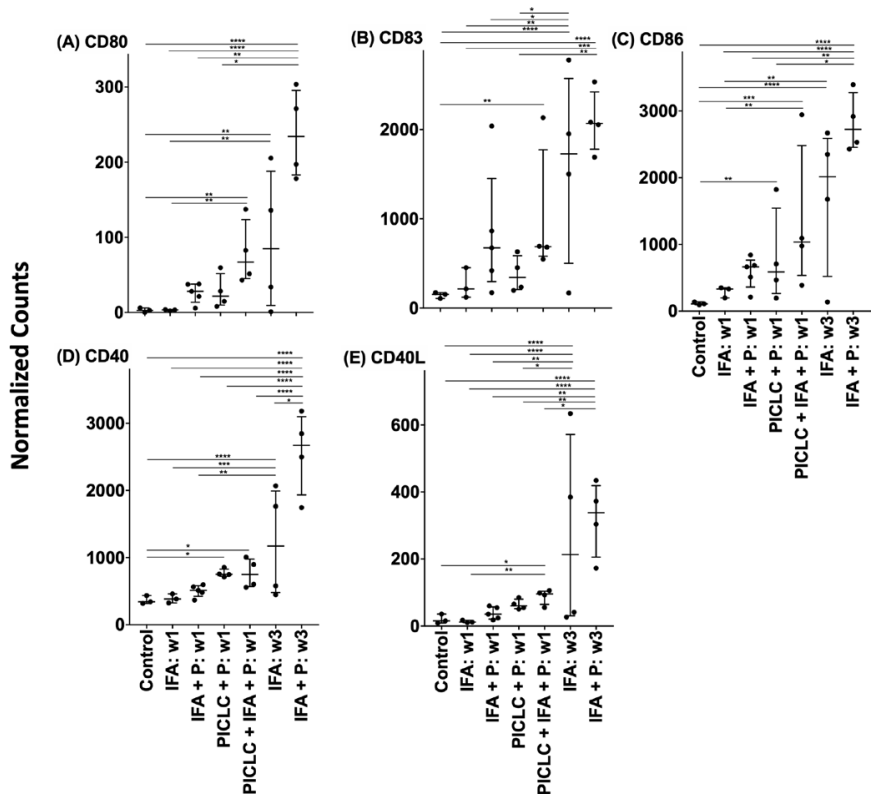

Figure 1 Expression of select genes within the vaccination site microenvironment following treatment with different vaccine compositions. Expression data are provided in terms of normalized counts. bars demonstrate median and IQR. $\mathrm{n}=27$. IFA, incomplete Freund's adjuvant; $\mathrm{P}$, peptide; w, week. ${ }^{*} \mathrm{P}<0.05,{ }^{\star *} \mathrm{p}<0.01,{ }^{\star \star *} \mathrm{p}<0.001,{ }^{* \star * *} \mathrm{p}<0.0001$. combination of adjuvants, when administered with peptides, support activation and maturation of DC in the VSME at 1 week (figure $1 \mathrm{~A}-\mathrm{C}$ ). Interestingly, there were significant increases in all these genes with $\mathrm{SSV} \times 3$ with IFA alone; however, these markers of DC activation and maturation were most dramatically upregulated after $\mathrm{SSV} \times 3$ with MELITAC 12.1 in IFA (figure $1 \mathrm{~A}-\mathrm{C}$ ) .

\section{CD40L and CD40 expression following single and repeat vaccination}

DC licensing is mediated through CD40 activation, and can be enhanced by addition of TLR agonists. ${ }^{32}$ Vaccination with a tetanus helper peptide (included in MELITAC 12.1) is effective for stimulating $\mathrm{CD}^{+} \mathrm{T}$-cell responses systemically $^{11}$; thus, we have hypothesized that these vaccines will activate CD4 T cells in the VSME, and that those CD4 T cells would express CD40L. We evaluated CD40 and CD40L expression in the VSME following both single and repeat immunization (figure $1 \mathrm{D}, \mathrm{E}$ ). One vaccine with peptides in pICLC, but not IFA, conferred an increase in CD40 expression, but not of CD40L. Both were increased at 1 week with polyICLC+IFA+peptides. $\mathrm{SSV} \times 3$ with IFA alone or in combination with peptides produced a dramatic increase in expression of both CD40 and CD40L ( $<<0.0001$ vs control, figure 1D,E). To verify that $\mathrm{CD} 40 \mathrm{~L}$ protein itself was present on CD4 T cells in the VSME, multiparameter immunofluorescence histology was performed on samples from two patients to assess co-expression of CD40L on $\mathrm{CD}^{+} \mathrm{CD}^{\text {neg }}$ cells as an estimate of $\mathrm{CD}^{+}$cells expressing CD40L protein in the VSME. (CD4 antibody staining was suboptimal; thus, $\mathrm{CD}^{+} \mathrm{CD}^{\text {neg }}$ staining was used as a surrogate for $\mathrm{CD} 4^{+}$ cells). A representative sample of the VSME after $\mathrm{SSV} \times 3$ with pICLC+IFA+peptides is shown in figure 2, which confirms expression of CD40L on multiple $\mathrm{CD}^{+} \mathrm{CD}^{\text {neg }}$ T cells.

\section{Transcription factors and Th1 dominance}

Having previously observed a dominance of GATA3 staining over Tbet staining within the VSME following 


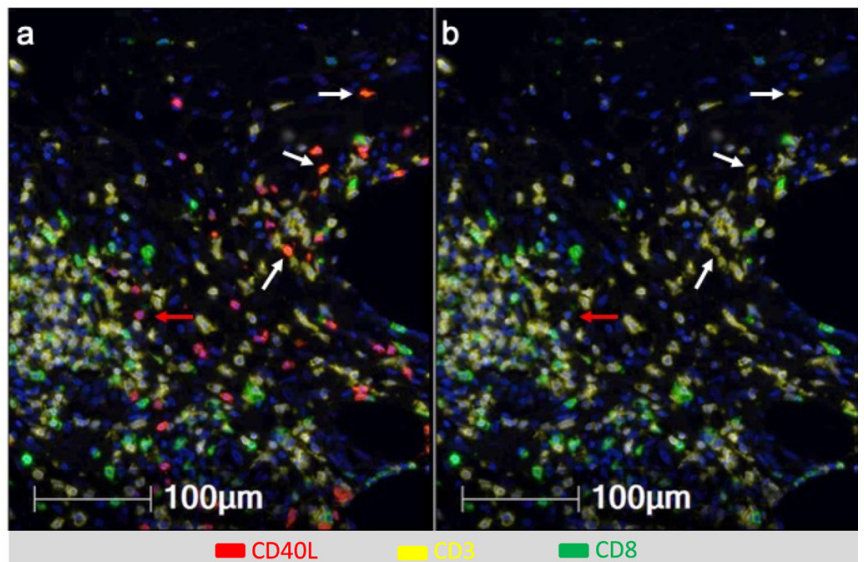

Figure 2 Multiparameter immunofluorescence histology of the vaccine site microenvironment postvaccination with peptides in IFA and pICLC showing co-expression of CD4OL and CD3 but not CD8. Selected markers include Dapi (blue), CD40L (red), CD3 (yellow), CD8 (green). (A) Includes CD40L and (B) excludes CD40L. White arrows depict $\mathrm{CD}^{+} \mathrm{CD}^{\text {neg }}$ T cells co-expressing CD40L and CD3 but not CD8. Red arrows depict cells expressing CD40L but not CD3 or CD8.

single injection with peptides and IFA, ${ }^{17}$ we conducted a more comprehensive evaluation of the expression of Th1, Th2, Th17 and Treg transcription factors (TBX21, GATA3, ROR $\gamma \mathrm{t} / \mathrm{RORC}$ and FOXP3, respectively), as well as Th1-associated genes for IFN $\gamma$ (IFNG) and STAT1, following vaccination. We hypothesized that repeat vaccination with peptide in IFA would enhances a Th1 signature compared with one vaccine, resulting in an increase in both STAT1 and IFNG expression.

One vaccine with IFA, alone or in combination with peptides, had little effect on expression of these genes. Vaccination with peptides in PICLC, with and without the addition of IFA, resulted in significant recruitment of $\mathrm{CD} 4^{+}$ and $\mathrm{CD}^{+} \mathrm{T}$ cells to the VSME as evidenced by increases in $\mathrm{CD} 4$ and CD8 expresssion (figure 3A,B). Peptides in pICLC, with and without IFA, also increased the expression of TBX21 and STAT1, but not IFNG (figure 3C-E) or any of the other transcription factors (figure 3). It was notable that there was baseline expression of GATA3, such that in normal skin, the Th2:Th1 ratios were high (299.34) (figure 3F). However, repeat $(\mathrm{SSV} \times 3)$ vaccination with peptides in IFA induced a dramatic increase in expression of Th1 genes, including TBX21 $(\mathrm{p}<0.001)$, STAT1 $(\mathrm{p}<0.01)$ and IFN $\gamma(\mathrm{p}<0.01)$, and a significant decrease in GATA3 and RORC compared with most other conditions (figure 3F,G). FOXP3 $(\mathrm{p}<0.01)$ expression also increased following repeat vaccination (figure $3 \mathrm{H}$ ). Interestingly, PD-1 expression remained relatively low in all conditions except in repeat $(\mathrm{SSV} \times 3)$ vaccination with IFA alone and peptide plus IFA (figure 3I). Additionally, LAG3 and TIM3 increased with repeat SSV with peptide plus IFA (figure 3J,K).
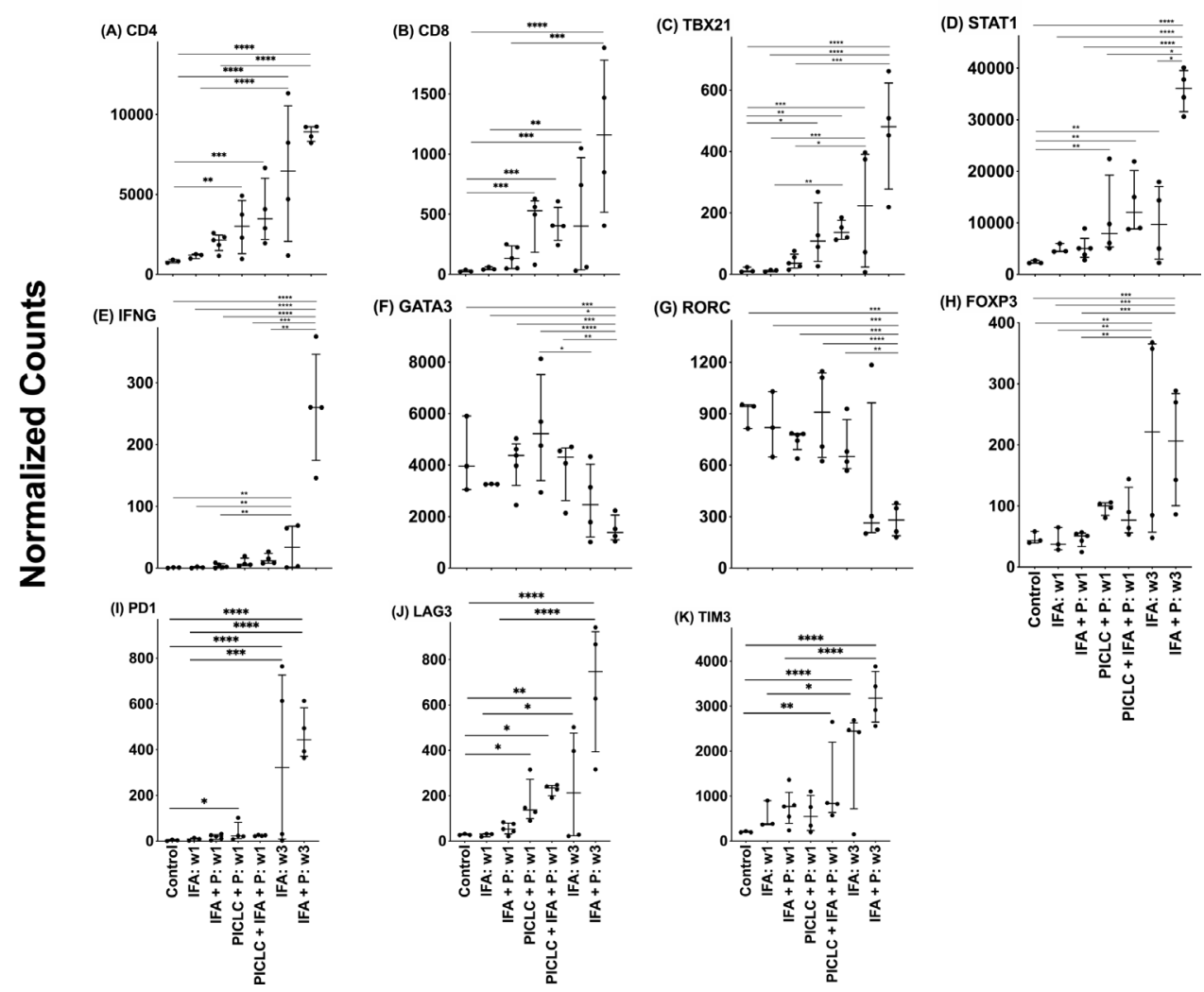

Figure 3 Expression of T-cell markers, transcription factors and markers of exhaustion within the vaccination site microenvironment following treatment with different vaccine compositions. Expression data are provided in terms of normalized counts. Bars demonstrate median and IQR. $\mathrm{n}=27$. IFA, incomplete Freund's adjuvant; $\mathrm{P}$, peptide; $\mathrm{w}, \mathrm{week}$. ${ }^{*} \mathrm{P}<0.05,{ }^{* *} \mathrm{p}<0.01$, ${ }^{* * *} \mathrm{p}<0.001,{ }^{* * *} \mathrm{p}<0.0001$. 


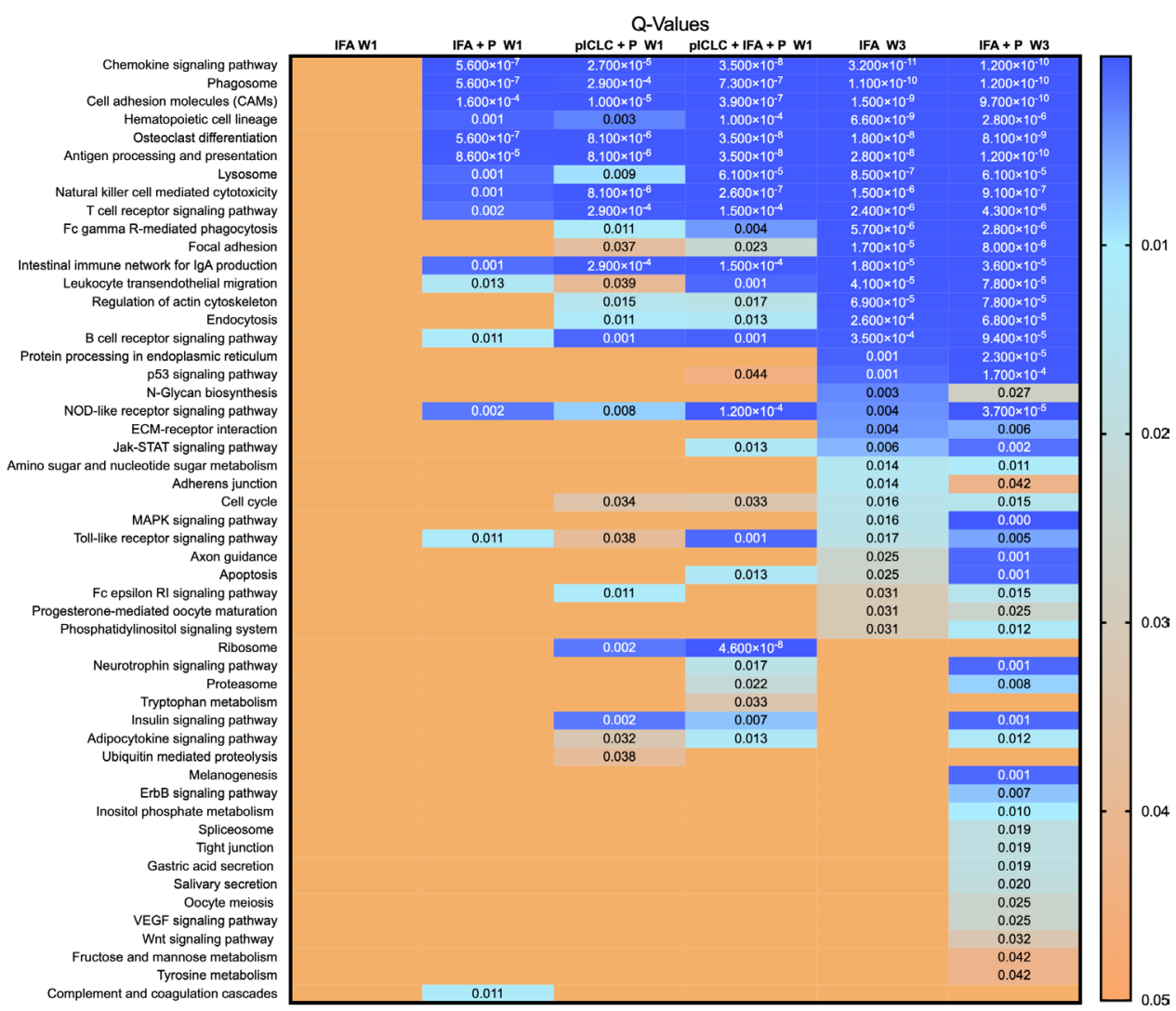

Figure 4 Results of GAGE pathway analysis performed on the complete set of differentially expressed genes under each vaccination condition. $Q$-values are provided for each enriched pathway under the various vaccination conditions. If a $Q$-value is not provided, the pathway was not enriched by the associated vaccination condition. IFA, incomplete Freund's adjuvant; $P$, peptide; w, week.

\section{GAGE pathway analysis}

To gain better insight into the biological pathways affected by the changes in the VSME, we applied GAGE pathway analysis on the complete set of differentially expressed genes under each vaccination condition. With the exception of single vaccination with IFA alone, all other treatment methods resulted in significant enrichment of 14 identical pathways, which were related to inflammation, chemokine signaling, T-cell and B-cell receptor signaling, antigen processing and presentation and cell migration (figure 4).

Overall, repeat vaccination with IFA affected a higher number of pathways than single vaccination, regardless of composition. SSV with the combination of peptides in IFA conferred the highest impact, leading to significant enrichment of 48 pathways. Most pathways impacted by the other vaccine combinations were included among those enriched by repeat vaccination with peptides in IFA. Exceptions include the 'Complement and Coagulation Cascade' pathway, which was significantly enriched only following single vaccination with peptides in IFA, the 'Ribosome' pathway, enriched following single vaccination with peptides in PICLC, with and without IFA, the 'Ubiquitin Mediated Proteolysis' pathway, affected by single vaccination with peptides in pICLC and the 'Tryptophan Metabolism' pathway, enriched only by single vaccination with peptides in PICLC and IFA.

\section{Arginase-1 expression following vaccination with pICLC and IFA}

Myeloid-derived suppressor cells can down-modulate antitumor immune responses ${ }^{33}$ with the potential to suppress T-cell responses and function, largely through the action of arginase-1 (ARG1). In an effort to characterize better the activity induced by peptide vaccines in IFA with or without polyICLC at the VSME, we analyzed the expression of ARG1 following single and repeat immunization (figure 5A). We found that one vaccine with IFA, either alone or in combination with peptide, has little effect on ARG1 expression. Following three vaccinations with peptide in IFA, expression of ARG1 trends downward and is nearly half that is observed within normal skin $(\mathrm{p}=0.06$, figure 5A). This gene expression is significantly lower than that seen with IFA and peptide at week one $(\mathrm{p}<0.01)$ or any other test condition except repeat vaccination with IFA alone. The results of a GSEA depict significant, global downregulation of the arginine and proline metabolism pathway following both single vaccination with peptides in pICLC (figure 6A) and repeat vaccination with peptides in IFA (figure 6B), compared with normal skin. ARG1 itself was not downregulated by peptides in pICLC (figure 6A) but highly downregulated by repeat vaccination with peptides in IFA (figure 6B). Taken together, these results suggest a qualitative difference in ARG1 expression following one vaccine with peptides in pICLC 

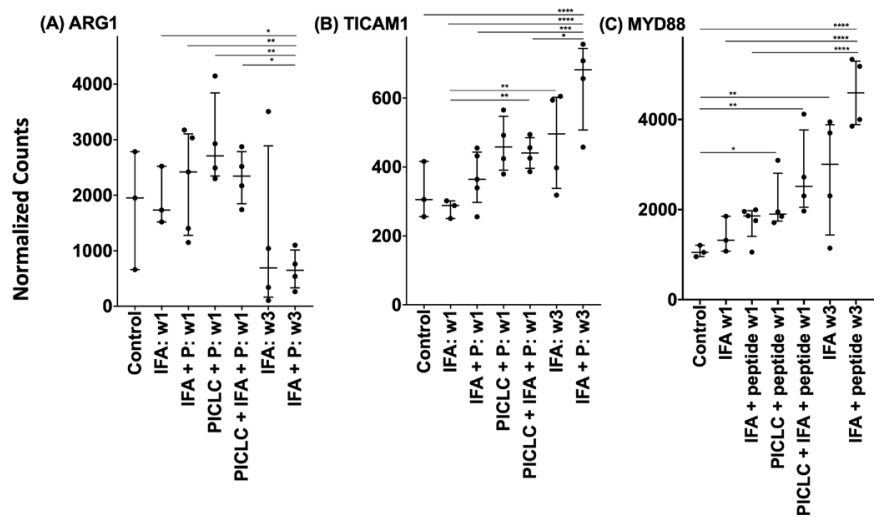

Figure 5 Expression of arginase-1 (A), TICAM1 (TRIF) (B) and MYD88 (C) within the vaccination site microenvironment following treatment with different vaccine compositions. Expression data are provided in terms of normalized counts. Bars demonstrate median and IQR. $\mathrm{n}=27$. IFA, incomplete Freund's adjuvant; $P$, peptide; w, week. ${ }^{*} P<0.05,{ }^{\star *} p<0.01$, ${ }^{\star * \star} p<0.001,{ }^{* \star \star \star} p<0.0001$.

compared with repeated vaccination with peptides in IFA (figure 5A) despite global downregulation of arginine and proline metabolism in both conditions.

\section{Toll-like receptor pathway signaling}

The GAGE pathway analysis revealed that the "toll-like receptor signaling' pathway was significantly perturbed by all vaccine conditions, with the exception of that involving single vaccination with IFA alone (figure 4). Furthermore, within the vaccine conditions that led to pathway enrichment, expression of both MYD88 and TRIF increased, and were within the top 500 most differently expressed genes, when compared with normal skin (data not shown). This was expected since polyICLC has
TLR3 agonist activity. However, it is notable that vaccination with peptides+IFAalso induced significant changes in TLR signaling. To understand these changes, we have evaluated changes in expression of the key adaptor molecules MYD88 and TRIF (TICAM1). TLR3 signals through TRIF, rather than MYD88; so, we expected TRIF expression to be enhanced when vaccinating with polyICLC. We found that TRIF expression trended higher 1 week after vaccination with polyICLC+peptides, with a significant increase when IFA was also in the vaccines (figure $5 \mathrm{~B}$ ). An unexpected finding was that $\mathrm{SSV} \times 3$ with peptides in IFA led to dramatic and significant increases in TRIF compared with most other conditions, despite there being no TLR agonist in those vaccine formulations.

There was also a significant rise in MYD88 expression following single vaccination with peptide in pICLC, compared with normal skin, and an even more significant enhancement in MYD88 was observed with peptide+polyICLC+IFA at 1 week (figure $5 \mathrm{C}$ ). Interestingly, SSV $\times 3$ with IFA alone also enhanced MYD88 expression. The most significant increases in MYD88 were observed with SSV with peptides in IFA $(\mathrm{p}<0.0001)$.

\section{Expression of a 12-chemokine gene signature associated with the formation of tertiary lymphoid structures}

In prior work, we identified TLS formation within the VSME after repeated vaccination with peptides in IFA. ${ }^{16} \mathrm{~A}$ 12-chemokine gene signature has been identified as being associated with TLS formation in melanoma ${ }^{34}$; so, we have evaluated this signature to understand possible contributing factors in TLS formation in the VSME. There was little effect on expression of these chemokine genes with one injection of IFA alone (online supplementary figure 2), either alone or in combination with peptide; however,

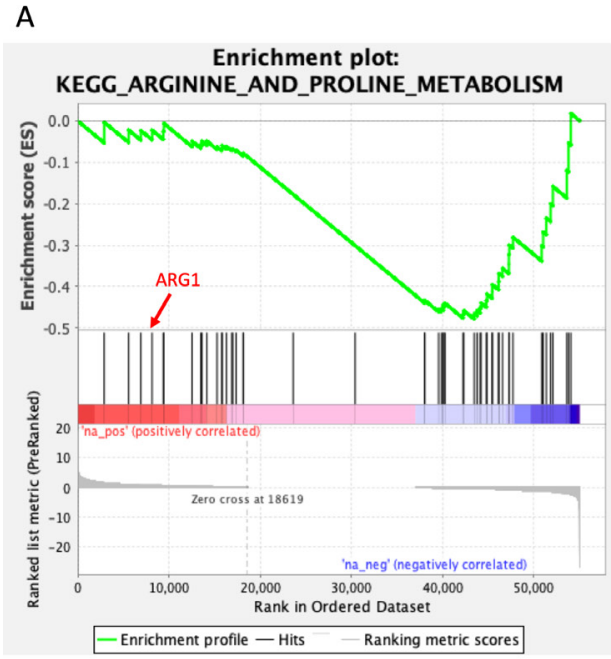

plCLC + P: W1 vs normal skin
B

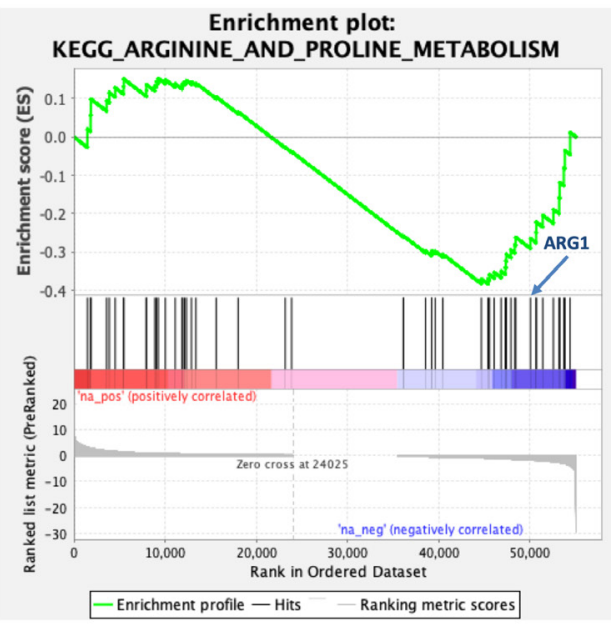

IFA + P: W3 vs normal skin

Figure 6 Gene set enrichment analysis highlighting the varied pattern of downregulation of arginine and proline metabolism following single vaccination with peptides in pICLC (A) and repeated vaccination with peptides in IFA (B). ARG1 is not meaningfully downregulated by peptides in pICLC (A) but is strongly downregulated in repeated vaccination with peptides in IFA (B) as shown by its position in the ranked list of genes. Both conditions were compared with normal skin control. Gene set enrichment data were generated using GAGE and included genes from the arginine and proline metabolism pathway. ARG1, arginase-1; IFA, incomplete Freund's adjuvant; pICLC, poly ICLC; $p$, peptide. 
one injection with peptides in pICLC led a significant increase in expression of 7/12 chemokine genes (CCL3, $\mathrm{p}<0.05$; CCL4, CXCL10, CXCL11, p<0.01; CCL5, CCL21, CXCL9, $\mathrm{p}<0.001)$. Vaccination with peptides, pICLC and IFA appeared to be the most effective combination after single injection, producing an increase in expression in 10/12 genes (CCL2, CCL3, CCL4, CCL5, CCL8, CXCL9, CXCL10, CXCL11, CCL18, CCL19). Notably, however, this combination did not increase expression of CXCL13, which has been strongly identified as playing a crucial role in TLS formation. In contrast, repeat vaccination with IFA, both alone and together with peptides, generated a dramatic increase in CXCL13 expression, plus an additional 9 (CCL2, CCL3, CCL4, CCL5, CCL8, CXCL9, CXCL10, CCL18, CCL19) or 10 (CCL2, CCL3, CCL4, CCL5, CCL8, CXCL9, CXCL10, CXCL11, CCL18, CCL19) genes, respectively.

\section{DISCUSSION}

A goal of vaccine therapy in cancer treatment is to produce strong, durable and antigen-specific T-cell responses. This requires effective vaccine adjuvants, the most optimal of which has yet to be determined. Current research has focused heavily on the use of TLR-agonistbased adjuvants, and the immunogenicity of these agents has been well-demonstrated in both mice and humans. ${ }^{35-39}$ The present study identifies effects of IFA, polyICLC and peptides in the VSME after one or three repeated vaccines, with evidence that SSV with IFA+peptide reduces the ARG1 expression, enhances CD40L expression and DC activation, supports a Th1-dominant VSME and induces a chemokine signature associated with formation of TLS.

IFA is a more traditional water-in-oil emulsion-based adjuvant. Its efficacy within melanoma vaccines has been called into question in recent murine studies, which reported that the adjuvant fosters the recruitment, exhaustion and eventual death of $\mathrm{T}$ cells within the vaccination site. ${ }^{40}$ Nevertheless, as we have reported, ${ }^{15}$ the applicability of those findings to humans may be limited by vaccine dosage volume and the extent of a native T-cell response. Methods in the prior murine study ${ }^{14}$ differed from these human trials by adoptive transfer of a large number of antigen-reactive $\mathrm{T}$ cells, as opposed to attempting to expand endogenous $\mathrm{T}$ cells, and by a much higher dose of IFA delivered per gram body weight (approximately 4 vs $0.01 \mu \mathrm{L}$ ). These and other factors may explain our different findings, which we have discussed in the primary report of the Mel58 clinical trial. ${ }^{15}$ Furthermore, recent human vaccine studies using IFA, conducted both within our laboratory and by others, have been promising, and have demonstrated the ability of IFA to generate a robust and antigen-specific CD8 T-cell response. ${ }^{10-12}$ In light of these data, we sought to understand possible mechanisms behind IFA's immunogenicity. Here, we focused on the cellular and molecular effects of IFA within the VSME. Our results highlight the ability of IFA to activate DCs and CD4 T cells within the VSME, support a Th1-dominant environment, and potentially reduce myeloid suppression within the vaccination site. We also present evidence suggesting that repeated SSV with IFA-containing vaccines might be more effective than single vaccination, including inducing the formation of TLS within the VSME.

Evidence of TLS within the VSME was previously demonstrated in a subset of participants on the Mel48 trial, by IHC. ${ }^{17}$ Our current data reinforce this notion, as repeated SSV with peptides in IFA significantly increased expression of 11 out of 12 chemokines whose overexpression has been associated with the TLS in melanoma. On the other hand, CCL21 has been defined as an important driver of TLS neogenesis, ${ }^{23}{ }^{41}$ but its expression was not consistently enhanced with IFA and peptides at week 3 .

In support of our previous IHC staining for cellular transcription factors, we found evidence in favor of repeated SSV with IFA generating a Th1-dominant microenvironment. After three vaccinations with IFA alone, expression of TBX21, STAT1 and IFNG all increased significantly, while expression of GATA3 and RORC trended downward. The addition of peptides enhanced these effects, causing a significant decrease in GATA3 and RORC expression compared with normal skin. This finding suggests that the VSME created by SSV with peptides in IFA may support activation and expansion of Th1 cells and cytotoxic $T$ cells. Interestingly, we also found that repeated SSV with peptides in IFA also upregulated PD-1, LAG3 and TIM3. Increased PD-1 may be explained by increased T-cell numbers and/or an increase of PD-1 expression by activated T cells in the VSME. However, taken together, the increase in these three genes may represent exhaustion on at least a subset of $T$ cells in the VSME. We have previously reported that $\mathrm{T}$ cells isolated from the VSME after SSV with peptides in IFA were less likely to produce IFN $\gamma$ in response to their cognate antigen than circulating T cells, ${ }^{18}$ which also suggests that some antigen-reactive T cells in the VSME are exhausted. However, questions remain about whether PD-1, LAG3 and TIM3 are co-expressed on the same $\mathrm{T}$ cells and whether exhausted cells represent a major or minor fraction of the antigen-reactive T cells in the VSME. Future single-cell and functional studies are needed in order to answer this question. The enhanced Th1 signatures, including IFN $\gamma$, suggest that immune activation remains a dominant feature of the VSME in this setting.

A question still remaining in light of our current work is whether repeated vaccination with peptides, in combination with both IFA and TLR agonists, will be more effective than repeated SSV with only IFA and peptides. As we reported previously, adding IFA to a single vaccine containing peptides in either of two TLR (lipopolysaccharide and pICLC), enhances the CD8 T-cell response rate and persistence compared with vaccines containing TLR agonist alone. ${ }^{15}$ It is possible that combining the two adjuvants in repeated vaccines will produce an even greater immune response. An ongoing clinical trial, Mel63 
(NCT02425306), will have samples to evaluate this question in patients receiving repeated SSV with TLR agonist, IFA and peptide.

Another interesting, yet unexpected, finding from our data was the fact that repeated SSV with IFA alone and in combination with peptides, induced marked increases in expression of both MyD88 and TRIF, despite the absence of an exogenous TLR agonist within the vaccine. There was also a dramatic and pervasive increase in signaling through the TLR pathway under all vaccination conditions. Activation of MyD88 and TRIF by the TLRs $1 / 2,2 / 6,5,7,8,9$ and TLR3, respectively, leads to downstream effects including release of cytokines and chemokines that support immune activation and cellular immunity. The activation of these signaling pathways by IFA, alone or in combination with peptides, sparks questions regarding their source of origin. It is possible that the signaling may be a direct effect of vaccination, itself. However, it is also conceivable that the skin microbiome may be responsible. A recent murine study demonstrated that vaccines involving IFA depend on MyD88 signaling for induction of humoral immunity. ${ }^{42}$ While the exact mechanism behind this process remains unknown, the authors of that study hypothesized a role for microbiotaderived signals. Specifically, tissue damage resulting from vaccination may expose commensal bacteria colonizing the surface of the skin and its appendages to TLRs, thus providing a potential trigger for the initiation of pathway signaling. Subsequent DC activation may then perpetuate this process. Future studies will focus on identifying skin microbiota and determining specific changes in TLR expression within the skin in order to understand these findings.

Notwithstanding the promise of TLR-based adjuvants and other novel adjuvants, the data in the present report support the continued evaluation of IFA with cancer vaccines, both as a single agent and in combination with TLR agonists. Despite its long history of use in cancer vaccines, little has been known about the cellular and molecular effects of IFA in the VSME. This study provides new data to understand the effects of vaccines incorporating IFA, and suggest prominent effects of repeated vaccines given at the same site. The finding of TLR signaling after SSV with peptides in IFA raises new questions about the impact that IFA has on the skin microbiota and the regulation of signaling pathways within the VSME, and supporting favorable effects of this adjuvant in humans.

\section{Author affiliations}

${ }^{1}$ Surgery, University of Virginia, Charlottesville, Virginia, USA

${ }^{2}$ Biology, University of Virginia, Charlottesville, Virginia, USA

${ }^{3}$ Office of Research Cores Administration (ORCA), University of Virginia School of Medicine, Charlottesville, Virginia, USA

${ }^{4}$ Public Health Sciences, University of Virginia School of Medicine, Charlottesville, Virginia, USA

${ }^{5}$ Biochemistry and Molecular Genetics, University of Virginia, Charlottesville, Virginia, USA

${ }^{6}$ Pathology, University of Virginia School of Medicine, Charlottesville, Virginia, USA
Acknowledgements The authors would like to thank Alex Hickman for her assistance in this work.

Contributors All authors contributed to the manuscript writing. KEP, MOM, KTL and CLSJ contributed to the study design and laboratory work. SJY and ISM performed the immunofluorescence histology. MMM performed the IHC and RNAseq correlation analyses. KS-C completed the RNAseq and the analyses were performed by PK, AFK and ST. KEP and MOM are co-first authors.

Funding NCI R01 CA057653 (CLS); T32 CA163177 (CLS, MM); P30 CA044579 (Biorepository and Tissue Research Facility, Genome Analysis and Technology Core, Bioinformatics Core: Molecular and Immunologic Translational Science Core); Clinical and Laboratory Integration Project (CLIP) grant from the Cancer Research Institute (CLS, MM), Rebecca Clary Harris Fellowship (MM), and gifts from Alice and Bill Goodwin and the Commonwealth Foundation for Cancer Research.

Competing interests CLSJ is listed as a co-inventor for some of the peptides used in the 12MP vaccine. The patents are held by the UVA Licensing and Ventures group (LVG).

Patient and public involvement statement Patients involved in this study signed informed consent for the clinical trial in which they participated. Both clinical trials from which samples were used were institutionally approved under IRB-HSR\# 13498 (Mel 48) and IRB-HSR\# 15781 (Mel 58).

\section{Patient consent for publication Not required.}

Provenance and peer review Not commissioned; externally peer reviewed.

Data availability statement Data are available on reasonable request.

Open access This is an open access article distributed in accordance with the Creative Commons Attribution 4.0 Unported (CC BY 4.0) license, which permits others to copy, redistribute, remix, transform and build upon this work for any purpose, provided the original work is properly cited, a link to the licence is given, and indication of whether changes were made. See https://creativecommons.org/ licenses/by/4.0/.

\section{ORCID iD}

Max 0 Meneveau http://orcid.org/0000-0002-8410-061X

\section{REFERENCES}

1 Leroux-Roels G. Unmet needs in modern vaccinology: adjuvants to improve the immune response. Vaccine 2010;28 Suppl 3:C25-36.

2 Stills HF. Adjuvants and antibody production: dispelling the myths associated with Freund's complete and other adjuvants. Ilar $J$ 2005;46:280-93.

3 Freund J, Thomson KJ, simple A. A simple, rapid technic of preparing water-in-oil emulsions of penicillin, drugs and biologics. Science 1945;101:468-9.

4 Atsmon J, Kate-llovitz E, Shaikevich D, et al. Safety and immunogenicity of multimeric-001--a novel universal influenza vaccine. J Clin Immunol 2012;32:595-603.

5 Atsmon J, Caraco Y, Ziv-Sefer S, et al. Priming by a novel universal influenza vaccine (Multimeric-001)-a gateway for improving immune response in the elderly population. Vaccine 2014;32:5816-23.

6 Pleguezuelos O, Robinson S, Stoloff GA, et al. Synthetic influenza vaccine (FLU-v) stimulates cell mediated immunity in a doubleblind, randomised, placebo-controlled phase I trial. Vaccine 2012;30:4655-60.

7 Pleguezuelos $\mathrm{O}$, Robinson S, Fernández A, et al. A synthetic influenza virus vaccine induces a cellular immune response that correlates with reduction in symptomatology and virus shedding in a randomized phase $\mathrm{lb}$ live-virus challenge in humans. Clin Vaccine Immunol 2015;22:828-35.

8 van Doorn E, Pleguezuelos O, Liu H, et al. Evaluation of the immunogenicity and safety of different doses and formulations of a broad spectrum influenza vaccine (FLU-v) developed by seek: study protocol for a single-center, randomized, double-blind and placebocontrolled clinical phase Ilb trial. BMC Infect Dis 2017;17:241.

9 Fox CB, Haensler J. An update on safety and immunogenicity of vaccines containing emulsion-based adjuvants. Expert Rev Vaccines 2013;12:747-58.

10 Slingluff CL, Petroni GR, Olson WC, et al. Effect of granulocyte/ macrophage colony-stimulating factor on circulating CD8+ and CD4+ T-cell responses to a multipeptide melanoma vaccine: outcome of a multicenter randomized trial. Clin Cancer Res 2009;15:7036-44.

11 Slingluff CL, Petroni GR, Chianese-Bullock KA, et al. Randomized multicenter trial of the effects of melanoma-associated helper 
peptides and cyclophosphamide on the immunogenicity of a multipeptide melanoma vaccine. J Clin Oncol 2011;29:2924-32.

12 Rosenberg SA, Sherry RM, Morton KE, et al. Tumor progression can occur despite the induction of very high levels of self/tumor antigen-specific $\mathrm{CD}^{+} \mathrm{T}$ cells in patients with melanoma. $\mathrm{J}$ Immunol 2005;175:6169-76.

13 Kenter GG, Welters MJP, Valentijn ARPM, et al. Vaccination against HPV-16 oncoproteins for vulvar intraepithelial neoplasia. $N$ Engl $J$ Med 2009;361:1838-47.

14 Hailemichael Y, Dai Z, Jaffarzad N, et al. Persistent antigen at vaccination sites induces tumor-specific CD8+ T cell sequestration, dysfunction and deletion. Nat Med 2013;19:465-72.

15 Melssen MM, Petroni GR, Chianese-Bullock KA, et al. A multipeptide vaccine plus Toll-like receptor agonists LPS or polyICLC in combination with incomplete Freund's adjuvant in melanoma patients. J Immunother Cancer 2019;7:163.

16 Harris RC, Chianese-Bullock KA, Petroni GR, et al. The vaccinesite microenvironment induced by injection of incomplete Freund's adjuvant, with or without melanoma peptides. J Immunother 2012;35:78-88.

17 Schaefer JT, Patterson JW, Deacon DH, et al. Dynamic changes in cellular infiltrates with repeated cutaneous vaccination: a histologic and immunophenotypic analysis. J Trans/ Med 2010;8:79.

18 Salerno EP, Shea SM, Olson WC, et al. Activation, dysfunction and retention of T cells in vaccine sites after injection of incomplete Freund's adjuvant, with or without peptide. Cancer Immunol Immunother 2013;62:1149-59.

19 Slingluff CL, Petroni GR, Chianese-Bullock KA, et al. Immunologic and clinical outcomes of a randomized phase II trial of two multipeptide vaccines for melanoma in the adjuvant setting. Clin Cancer Res 2007:13:6386-95

20 Caux C, Massacrier C, Vanbervliet B, et al. Activation of human dendritic cells through CD40 cross-linking. J Exp Med 1994;180:1263-72.

21 Cella M, Scheidegger D, Palmer-Lehmann K, et al. Ligation of CD40 on dendritic cells triggers production of high levels of interleukin-12 and enhances T cell stimulatory capacity: T-T help via APC activation. J Exp Med 1996;184:747-52.

22 Messina JL, Fenstermacher DA, Eschrich S, et al. 12-Chemokine gene signature identifies lymph Node-like structures in melanoma: potential for patient selection for immunotherapy? Sci Rep 2012;2.

23 Ciccia F, Rizzo A, Maugeri R, et al. Ectopic expression of CXCL13, BAFF, April and LT- $\beta$ is associated with artery tertiary lymphoid organs in giant cell arteritis. Ann Rheum Dis 2017;76:235-43.

24 Slingluff CL, Yamshchikov G, Neese P, et al. Phase I trial of a melanoma vaccine with gp100(280-288) peptide and tetanus helper peptide in adjuvant: immunologic and clinical outcomes. Clin Cancer Res 2001;7:3012-24.

25 Patro R, Duggal G, Love Ml, et al. Salmon provides fast and bias-aware quantification of transcript expression. Nat Methods $2017 ; 14: 417-9$

26 Soneson C, Love MI, Robinson MD. Differential analyses for RNASeq: transcript-level estimates improve gene-level inferences. F1000Res 2015;4:1521.
27 Anders S, Huber W. Differential expression analysis for sequence count data. Genome Biol 2010;11:R106.

28 Love MI, Huber W, Anders S. Moderated estimation of fold change and dispersion for RNA-Seq data with DESeq2. Genome Biol 2014; 15:550.

29 Luo W, Friedman MS, Shedden K, et al. Gage: generally applicable gene set enrichment for pathway analysis. BMC Bioinformatics 2009;10:161.

30 Kanehisa M, Goto S, Sato Y, et al. Data, information, knowledge and principle: back to metabolism in KEGG. Nucleic Acids Res 2014;42:D199-205.

31 Subramanian A, Tamayo P, Mootha VK, et al. Gene set enrichment analysis: a knowledge-based approach for interpreting genome-wide expression profiles. Proc Natl Acad Sci U S A 2005;102:15545-50.

32 Ahonen CL, Doxsee CL, McGurran SM, et al. Combined TLR and CD40 triggering induces potent CD8+ T cell expansion with variable dependence on type I IFN. J Exp Med 2004;199:775-84.

33 Fernández A, Oliver L, Alvarez R, et al. Adjuvants and myeloidderived suppressor cells: enemies or allies in therapeutic cancer vaccination. Hum Vaccin Immunother 2014;10:3251-60.

34 Messina JL, Fenstermacher DA, Eschrich S, et al. 12-Chemokine gene signature identifies lymph node-like structures in melanoma: potential for patient selection for immunotherapy? Sci Rep 2012;2:765

35 Sanchez PJ, McWilliams JA, Haluszczak C, et al. Combined TLR/CD40 stimulation mediates potent cellular immunity by regulating dendritic cell expression of CD70 in vivo. $J$ Immunol 2007;178:1564-72

36 Lapteva N, Seethammagari MR, Hanks BA, et al. Enhanced activation of human dendritic cells by inducible CD40 and Toll-like receptor-4 ligation. Cancer Res 2007:67:10528-37.

37 Carpenter EL, Mick R, Rüter J, et al. Activation of human B cells by the agonist CD40 antibody CP-870,893 and augmentation with simultaneous Toll-like receptor 9 stimulation. $J$ Transl Med 2009;7:765.

38 Ahonen CL, Doxsee CL, McGurran SM, et al. Combined TLR and CD40 triggering induces potent CD8+ T cell expansion with variable dependence on type I IFN. J Exp Med 2004;199:775-84.

39 Speiser DE, Liénard D, Rufer N, et al. Rapid and strong human $\mathrm{CD} 8+\mathrm{T}$ cell responses to vaccination with peptide, IFA, and CpG oligodeoxynucleotide 7909. J Clin Invest 2005;115:739-46.

40 Hailemichael Y, Dai Z, Jaffarzad N, et al. Persistent antigen at vaccination sites induces tumor-specific $C D 8^{+} T$ cell sequestration, dysfunction and deletion. Nat Med 2013;19:465-72.

41 Manzo A, Paoletti S, Carulli M, et al. Systematic microanatomical analysis of CXCL13 and CCL21 in situ production and progressive lymphoid organization in rheumatoid synovitis. Eur $\mathrm{J}$ Immunol 2005;35:1347-59.

42 Riteau N, Radtke AJ, Shenderov K, et al. Water-in-Oil-Only adjuvants selectively promote $\mathrm{T}$ follicular helper cell polarization through a type I IFN and IL-6-dependent pathway. J Immunol 2016;197:3884-93. 